\title{
A new 2-D multi-stable chaotic attractor and its MultiSim electronic circuit design
}

\author{
Sundarapandian Vaidyanathan ${ }^{1}$, Aceng Sambas ${ }^{2}$, Mohamad Afendee Mohamed ${ }^{3}$, \\ Mustafa Mamat $^{3}$, W. S. Mada Sanjaya ${ }^{4}$, Sudarno $^{5}$ \\ ${ }^{1}$ Research and Development Centre, Vel Tech University, Chennai, Tamil Nadu, India \\ ${ }^{2}$ Department of Mechanical Engineering, Universitas Muhammadiyah Tasikmalaya, Indonesia \\ ${ }^{3}$ Faculty of Informatics and Computing, Universiti Sultan Zainal Abidin, KualaTerengganu, Malaysia \\ ${ }^{4}$ Departement of Physics, UIN Sunan Gunung Djati Bandung, Indonesia \\ ${ }^{5}$ Faculty of Engineering, Universitas Muhammadiyah Ponorogo, Indonesia
}

\begin{tabular}{l}
\hline Article Info \\
\hline Article history: \\
Received Aug 26, 2020 \\
Revised Mar 6, 2021 \\
Accepted Mar 17, 2021 \\
\hline
\end{tabular}

Keywords:

Chaos

Chaotic system

Electronic circuit

Integral sliding control

\begin{abstract}
A new multi-stable system with a double-scroll chaotic attractor is developed in this paper. Signal plots are simulated using MATLAB and multi-stability is established by showing two different coexisting double-scroll chaotic attractors for different states and same set of parameters. Using integral sliding control, synchronized chaotic attractors are achieved between driveresponse chaotic attractors. A MultiSim circuit is designed for the new chaotic attractor, which is useful for practical engineering realizations.
\end{abstract}

This is an open access article under the CC BY-SA license.

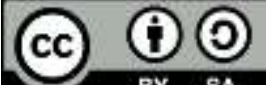

\section{Corresponding Author:}

Mohamad Afendee Mohamed

Faculty of Informatics and Computing

Universiti Sultan Zainal Abidin

KualaTerengganu, Malaysia

Email: mafendee@unisza.edu.my

\section{INTRODUCTION}

Chaotic dynamical models with double-scroll attractors have been analyzed in the science literature [1], [2]. These attractors resemble like butterfly wings due to their double-scroll shape. Especially, the dynamical plants exhibiting multi-stability and cohappening chaotic attractors have been studied [3], [4]. Engineering fields have many utilizations of chaotic attractors [2]. Some common utilizations are enlisted such as oscillations [5], [6], vibrations [7], neuron models [8], [9], control and memristor models [10]-[12], mechanical attractors [13], [14].

In the control literature, there are many control techniques available for the control and synchronization of chaotic systems [2]. Bahoo and Poria [13] used active control method for food chain model. Mustafa et al. [14] used chaos-enhanced cuckoo search for economic dispatch with valve point effects. Vaidyanathan and Rasappan [15] used active control for the hybrid synchronization of hyperchaotic Qi and Lü systems. Vaidyanathan [16] used active control for stabilizing the state trajectories of a new hyperchaotic system with three quadratic nonlinearities. Medhaffar et al. [17] investigated the stabilization of unstable periodic orbits of continuous time chaotic systems using adaptive fuzzy controllers. Boubellouta and 
Boulkroune [18] investigated the problem of chaos synchronization based on fractional-order intelligent sliding-mode control approach for a class of fractional-order chaotic optical systems with unknown dynamics and disturbances. Vaidyanathan [19] studied the global chaos synchronization of Tokamak chaotic systems with symmetric and magnetically confined plasma. Khan and Kumar [20] studied the T-S fuzzy observed based design and synchronization of chaotic and hyper-chaotic dynamical systems.

The novelty of this work is the modelling a new double-scroll chaotic attractor with interesting dynamic properties. The signal plots, dynamical properties and multi-stability with cohappening chaotic attractors are reported for the new chaotic attractor. For practical realizations, an electronic circuit is immensely useful after the modelling of a new chaotic attractor [21]-[26]. A MultiSim electronic circuit model of the new chaotic attractor is carried out and a good match between the MultiSim circuit outputs and the MATLAB signal plots has been found.

\section{A NEW DOUBLE-SCROLL MULTI-STABLE CHAOTIC ATTRACTOR}

We first give the dynamics of a new system described as follows:

$$
\left\{\begin{array}{l}
\dot{p}_{1}=\alpha\left(p_{2}-p_{1}\right)+p_{2} p_{3} \\
\dot{p}_{2}=\beta p_{2}-p_{1} p_{3} \\
\dot{p}_{3}=p_{1} p_{2}-\gamma p_{3}+\delta\left|p_{2}\right|
\end{array}\right.
$$

We note that $\Lambda=(\alpha, \beta, \gamma, \delta)$ is the parameter and $P=\left(p_{1}, p_{2}, p_{3}\right)$ is the phase vector. Using Wolf's approach [27], we will show that the model (1) will exhibit a chaotic attractor for

$$
\Lambda=(\alpha, \beta, \gamma, \delta)=(40,26,5,0.2)
$$

For MATLAB simulations, the initial phase vector is chosen as $P(0)=(0.1,0.3,0.2)$.

Then the Lyapunov indices of (1) are estimated using Wolf's approach [27] as follows:

$$
L E_{1}=3.9125, L E_{2}=0, L E_{3}=-22.9125
$$

Using (3), it is concluded that the model (1) has chaoticity and dissipativity.

The double-scroll attractor of the model (1) is simulated in various planes in Figure 1.

The balance points of the new double-scroll attractor (1) for $(\alpha, \beta, \gamma, \delta)=(40,26,5,0.2)$ are calculated as below:

$$
P_{0}=\left[\begin{array}{l}
0 \\
0 \\
0
\end{array}\right], \quad P_{1}=\left[\begin{array}{c}
11.3022 \\
7.8017 \\
17.9473
\end{array}\right], P_{2}=\left[\begin{array}{c}
-11.3022 \\
-7.8017 \\
17.9473
\end{array}\right]
$$

By finding spectral values of the linearization matrices of the double-scroll system (1), it can be ascertained that the balance point $P_{0}$ is a saddle point, and $P_{1}, P_{2}$ are saddle-foci.

We next demonstrate that the new double-scroll system (1) has cohappening chaotic attractors.

When selecting $(\alpha, \beta, \gamma, \delta)=(40,26,5,0.2)$, and the initial phase vectors $P_{0}=(0.1,0.3,0.2)$ (blue) and $Q_{0}=(-0.5,-0.3,-0.5)$ (red), the new double-scroll chaotic attractor (1) depicts cohappening chaotic attractor (blue) and chaotic attractor (red) as plotted in Figure 2. 


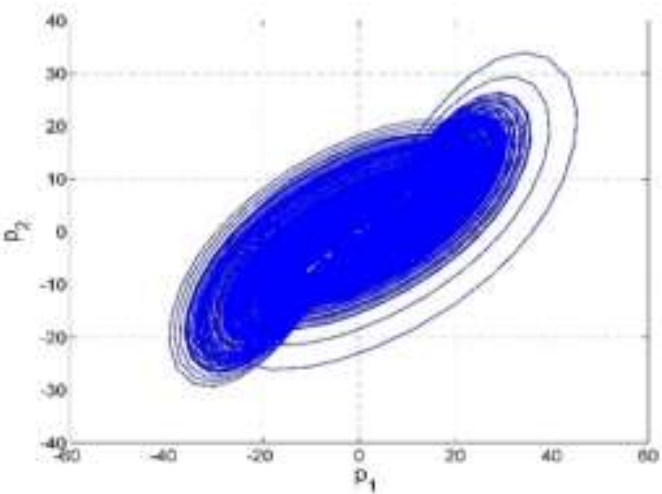

(a)

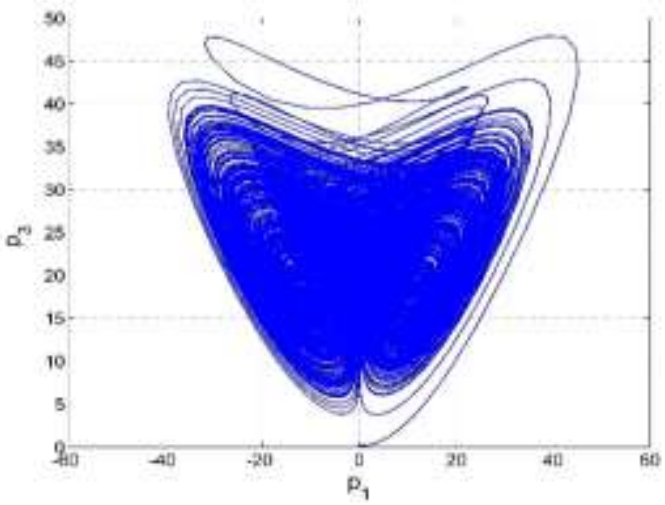

(c)

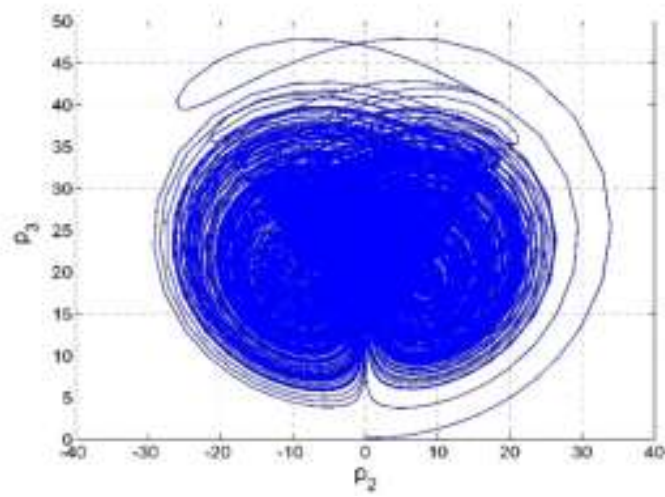

(b)

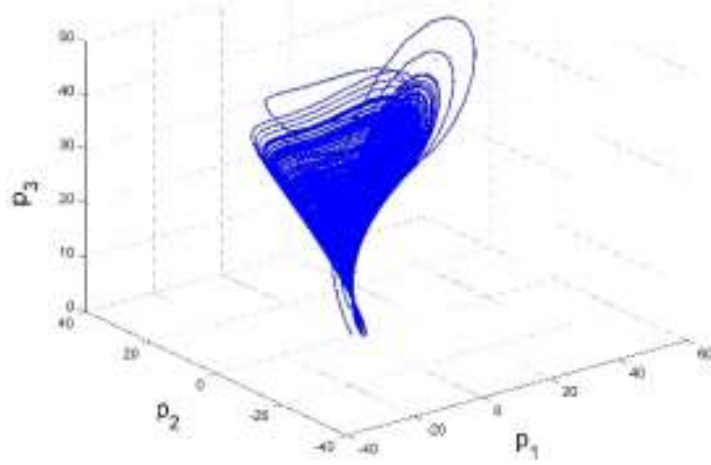

(d)

Figure 1. MATLAB phase plots showing double-scroll chaotic attractor of the model (1)

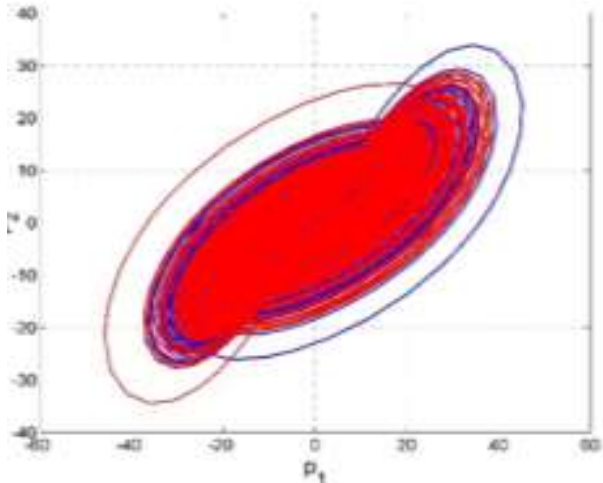

(a)

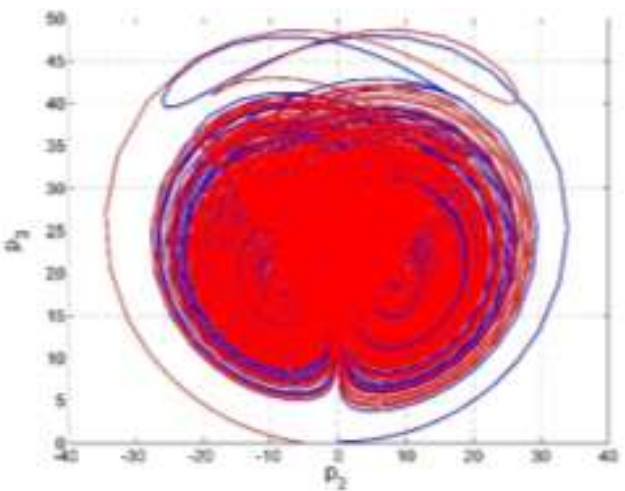

(b)

Figure 2. Multi-stability of the new double-scroll attractor (1): Cohappening chaotic attractors

\section{INTEGRAL SLIDING CONTROL DESIGN FOR THE PHASE SYNCHRONIZATION OF DOUBLE-SCROLL CHAOTIC ATTRACTORS}

For the phase synchronization of double-scroll chaotic attractor, we consider a pair of drive-response chaotic attractors listed as follows.

$$
\left\{\begin{array}{l}
\dot{p}_{1}=\alpha\left(p_{2}-p_{1}\right)+p_{2} p_{3} \\
\dot{p}_{2}=\beta p_{2}-p_{1} p_{3} \\
\dot{p}_{3}=p_{1} p_{2}-\gamma p_{3}+\delta\left|p_{2}\right|
\end{array}\right.
$$


$\left\{\begin{array}{l}\dot{q}_{1}=\alpha\left(q_{2}-q_{1}\right)+q_{2} q_{3}+v_{1} \\ \dot{q}_{2}=\beta q_{2}-q_{1} q_{3}+v_{2} \\ \dot{q}_{3}=q_{1} q_{2}-\gamma q_{3}+\delta\left|q_{2}\right|+v_{3}\end{array}\right.$

The phase synchronization error between (5) and (6) can be defined as below:

$\left\{\begin{array}{l}\varepsilon_{1}=q_{1}-p_{1} \\ \varepsilon_{2}=q_{2}-p_{2} \\ \varepsilon_{3}=q_{3}-p_{3}\end{array}\right.$

A simple calculation pinpoints the dynamics of the phase synchronization error as below:

$$
\left\{\begin{array}{l}
\dot{\varepsilon}_{1}=\alpha\left(\varepsilon_{2}-\varepsilon_{1}\right)+q_{2} q_{3}-p_{2} p_{3}+v_{1} \\
\dot{\varepsilon}_{2}=\beta \varepsilon_{2}-q_{1} q_{3}+p_{1} p_{3}+v_{2} \\
\dot{\varepsilon}_{3}=-\gamma \varepsilon_{3}+q_{1} q_{2}-p_{1} p_{2}+\delta\left(\left|q_{2}\right|-\left|p_{2}\right|\right)+v_{3}
\end{array}\right.
$$

We define the integral sliding surface associated with each error variable as below:

$$
\left\{\begin{array}{l}
z_{1}=\left[\frac{d}{d t}+\psi_{1}\right]\left[\int_{0}^{t} \varepsilon_{1}(\tau) d \tau\right]=\varepsilon_{1}+\psi_{1} \int_{0}^{t} z_{1}(\tau) d \tau \\
z_{2}=\left[\frac{d}{d t}+\psi_{2}\right]\left[\int_{0}^{t} \varepsilon_{2}(\tau) d \tau\right]=\varepsilon_{2}+\psi_{2} \int_{0}^{t} z_{2}(\tau) d \tau \\
z_{3}=\left[\frac{d}{d t}+\psi_{3}\right]\left[\int_{0}^{t} \varepsilon_{3}(\tau) d \tau\right]=\varepsilon_{3}+\psi_{3} \int_{0}^{t} z_{3}(\tau) d \tau
\end{array}\right.
$$

Taking time-derivative of all the of (9), we obtain as below:

$$
\left\{\begin{array}{l}
\dot{z}_{1}=\dot{\varepsilon}_{1}+\psi_{1} \varepsilon_{1} \\
\dot{z}_{2}=\dot{\varepsilon}_{2}+\psi_{2} \varepsilon_{2} \\
\dot{z}_{3}=\dot{\varepsilon}_{3}+\psi_{3} \varepsilon_{3}
\end{array}\right.
$$

We take $\psi_{1}, \psi_{2}, \psi_{3}$ as positive constants.

Next, we set the dynamics of the sliding variables as follows:

$$
\left\{\begin{array}{l}
\dot{z}_{1}=-\theta_{1} \operatorname{sgn}\left(z_{1}\right)-\mu_{1} z_{1} \\
\dot{z}_{2}=-\theta_{2} \operatorname{sgn}\left(z_{2}\right)-\mu_{2} z_{2} \\
\dot{z}_{3}=-\theta_{3} \operatorname{sgn}\left(z_{3}\right)-\mu_{3} z_{3}
\end{array}\right.
$$

From (10) and (11), we deduce the following:

$$
\left\{\begin{array}{l}
\dot{e}_{1}+\lambda_{1} e_{1}=-\varphi_{1} \operatorname{sgn}\left(s_{1}\right)-k_{1} s_{1} \\
\dot{e}_{2}+\lambda_{2} e_{2}=-\varphi_{2} \operatorname{sgn}\left(s_{2}\right)-k_{2} s_{2} \\
\dot{e}_{3}+\lambda_{3} e_{3}=-\varphi_{3} \operatorname{sgn}\left(s_{3}\right)-k_{3} s_{3}
\end{array}\right.
$$

Combining (8) and (12), we obtain the following:

$$
\left\{\begin{array}{l}
\alpha\left(\varepsilon_{2}-\varepsilon_{1}\right)+q_{2} q_{3}-p_{2} p_{3}+v_{1}+\psi_{1} \varepsilon_{1}=-\theta_{1} \operatorname{sgn}\left(z_{1}\right)-\mu_{1} z_{1} \\
\beta \varepsilon_{2}-q_{1} q_{3}+p_{1} p_{3}+v_{2}+\psi_{2} \varepsilon_{2}=-\theta_{2} \operatorname{sgn}\left(z_{2}\right)-\mu_{2} z_{2} \\
-\gamma \varepsilon_{3}+q_{1} q_{2}-p_{1} p_{2}+\delta\left(\left|q_{2}\right|-\left|p_{2}\right|\right)+v_{3}+\psi_{3} e_{3}=-\theta_{3} \operatorname{sgn}\left(z_{3}\right)-\mu_{3} z_{3}
\end{array}\right.
$$


The integral sliding controls are deduced from (13) as below:

$$
\left\{\begin{array}{l}
v_{1}=-\alpha\left(\varepsilon_{2}-\varepsilon_{1}\right)-q_{2} q_{3}+p_{2} p_{3}-\psi_{1} \varepsilon_{1}-\theta_{1} \operatorname{sgn}\left(z_{1}\right)-\mu_{1} z_{1} \\
v_{2}=-\beta e_{2}+q_{1} q_{3}-p_{1} p_{3}-\psi_{2} \varepsilon_{2}-\theta_{2} \operatorname{sgn}\left(z_{2}\right)-\mu_{2} z_{2} \\
v_{3}=\gamma e_{3}-q_{1} q_{2}+p_{1} p_{2}-\delta\left(\left|q_{2}\right|-\left|p_{2}\right|\right)-\psi_{3} \varepsilon_{3}-\theta_{3} \operatorname{sgn}\left(z_{3}\right)-\mu_{3} z_{3}
\end{array}\right.
$$

Theorem 1. The integral sliding control law defined by (14) achieves the global phase chaos synchronization between the new double-scroll attractors (5) and (6), where the constants $\psi_{i}, \theta_{i}, \mu_{i}$, $(i=1,2,3)$ are all positive.

Proof. First, as a positive definite Liapunov function candidate, we choose the function

$$
W\left(z_{1}, z_{2}, z_{3}\right)=0.5\left(z_{1}^{2}+z_{2}^{2}+z_{3}^{2}\right)
$$

We calculate the time-derivative of $W$ as below:

$$
\dot{W}=z_{1} \dot{z}_{1}+z_{2} \dot{z}_{2}+z_{3} \dot{z}_{3}
$$

Combining (11) and (16), we get

$$
\dot{W}=z_{1}\left[-\theta_{1} \operatorname{sgn}\left(z_{1}\right)-\mu_{1} z_{1}\right]+z_{2}\left[-\theta_{2} \operatorname{sgn}\left(z_{2}\right)-\mu_{2} z_{2}\right]+z_{3}\left[-\theta_{3} \operatorname{sgn}\left(z_{3}\right)-\mu_{3} z_{3}\right]
$$

Simplifying (17), we obtain

$$
\dot{W}=-\theta_{1}\left|z_{1}\right|-\mu_{1} z_{1}^{2}-\theta_{2}\left|z_{2}\right|-\mu_{2} z_{2}^{2}-\theta_{3}\left|z_{3}\right|-\mu_{3} z_{3}^{2}
$$

Since $\theta_{1}, \theta_{2}, \theta_{3}>0$ and $\mu_{1}, \mu_{2}, \mu_{3}>0$, we see that $\dot{W}$ is a negative definite function.

From Liapunov stability theory [28], we find $\left(z_{1}(t), z_{2}(t), z_{3}(t)\right) \rightarrow(0,0,0)$ as $t \rightarrow \infty$.

Hence, we observe that $\left(\varepsilon_{1}(t), \varepsilon_{2}(t), \varepsilon_{3}(t)\right) \rightarrow(0,0,0)$ as $t \rightarrow \infty$.

For MATLAB simulations, we assume the parameter vector as in the chaotic case, viz. $(\alpha, \beta, \gamma, \delta)=(40,26,5,0.2)$. We also assume the gains as $\psi_{i}=0.1, \theta_{i}=0.1$, and $\mu_{i}=20$ for $i=1,2,3$.

The initial state of the drive system $(5)$ is picked as $\left(p_{1}(0), p_{2}(0), p_{3}(0)\right)=(3,-0.5,2)$ and the initial state of the response system $(6)$ is picked as $\left(q_{1}(0), q_{2}(0), q_{3}(0)\right)=(1.5,0.9,4.2)$.

Figure 3 shows the phase synchronization error between systems (5) and (6).

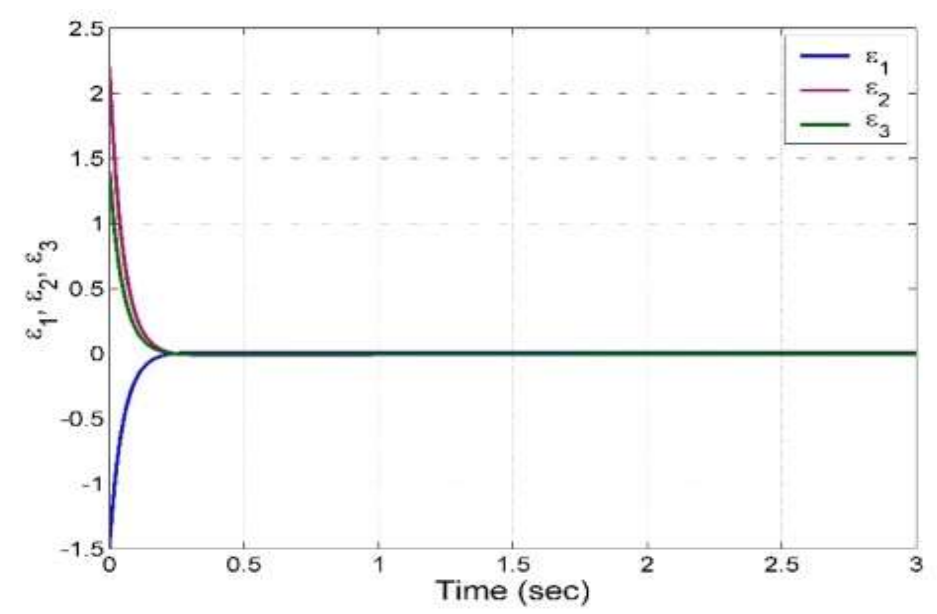

Figure 3. The phase synchronization error between the systems (5) and (6) 


\section{MULTISIM CIRCUIT DESIGN OF THE NEW DOUBLE-SCROLL ATTRACTOR}

The MultiSim electronic circuit of the new double-scroll attractor (1) is realized by using off-theshelf components such as resistors, capacitors, operational amplifiers and analog multipliers. The phases $p_{1}$, $p_{2}, p_{3}$ of the double-scroll attractor (1) are the voltages across the capacitors $C_{1}, C_{2}$ and $C_{3}$, respectively. The electronic circuit of the new double-scroll attractor is realized in MultiSim by 19 resistors, 8 operational amplifiers (TL082CD), 3 multipliers (AD633JN), 2 diodes (1N4148) and 3 capacitors. By the use of Kirchhoff's circuit laws into the circuit in Figure 4, its circuital equations are obtained as (19):

$$
\left\{\begin{array}{l}
\dot{p}_{1}=\frac{1}{C_{1} R_{1}} p_{2}-\frac{1}{C_{1} R_{2}} p_{1}+\frac{1}{10 C_{1} R_{3}} p_{2} p_{3} \\
\dot{p}_{2}=\frac{1}{C_{2} R_{4}} p_{2}-\frac{1}{10 C_{2} R_{5}} p_{1} p_{3} \\
\dot{p}_{3}=\frac{1}{10 C_{3} R_{6}} p_{1} p_{2}-\frac{1}{C_{3} R_{7}} p_{3}+\frac{1}{C_{3} R_{8}}\left|p_{2}\right|
\end{array}\right.
$$

We selected $R_{1}=R_{2}=10 \mathrm{k} \Omega, R_{3}=R_{5}=R_{6}=40 \mathrm{k} \Omega, R_{4}=15.384 \mathrm{k} \Omega, R_{7}=80 \mathrm{k} \Omega, R_{8}=2 \mathrm{M} \Omega, R_{9}=$ $R_{10}=R_{11}=R_{12}=R_{13}=R_{14}=R_{15}=R_{16}=R_{17}=R_{18}=R_{19}=100 \mathrm{k} \Omega, C_{1}=C_{2}=C_{3}=3.2 \mathrm{nF}$.

Figures 5-7 with MultiSim outputs of the double-scroll chaotic attractor (19) exhibit a good match with the MATLAB outputs of the double-scroll chaotic attractor (1) shown in Figure 1.

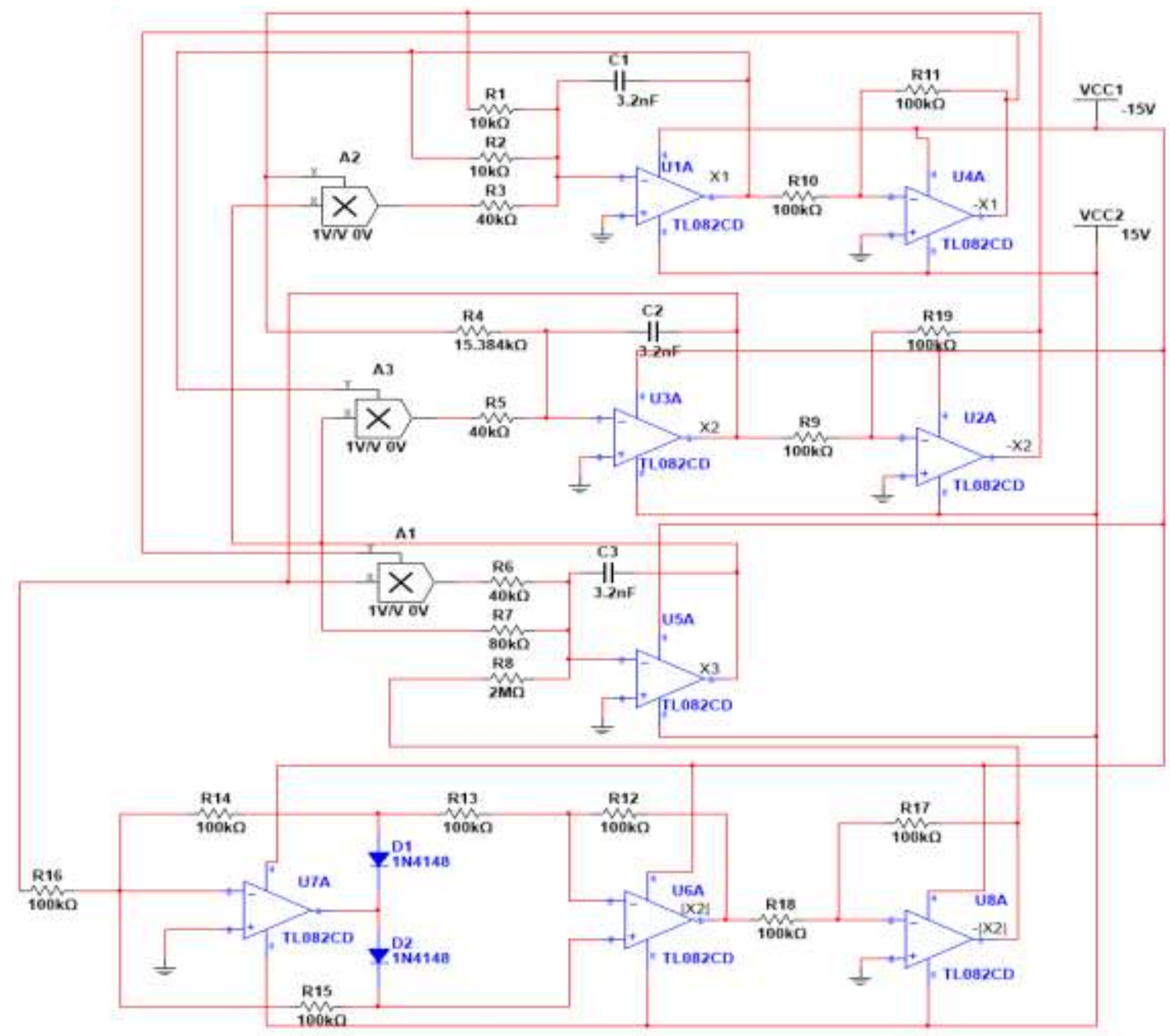

Figure 4. The circuit schematic of the double-scroll chaotic attractor (19) (Note: $p_{1}, p_{2}, p_{3}=x_{1}, x_{2}, x_{3}$ ) 


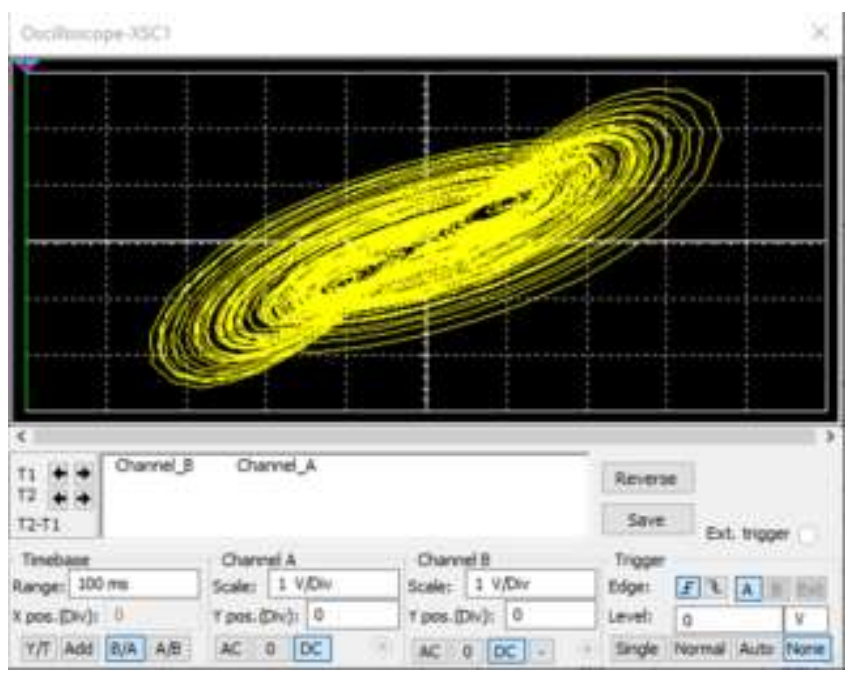

Figure 5. MultiSim output of the double-scroll circuit (19) in $\left(p_{1}, p_{2}\right)$ - plane

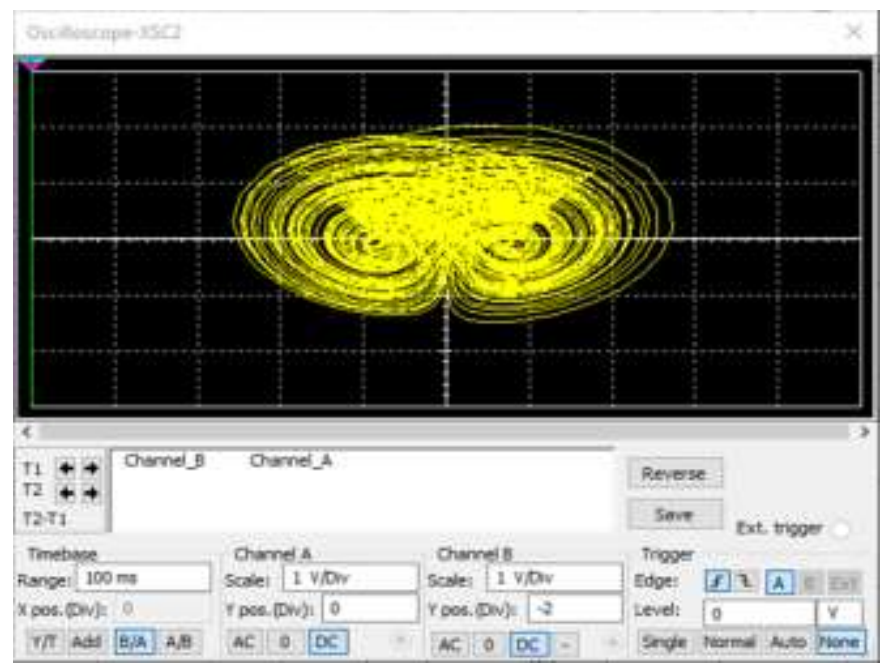

Figure 6. MultiSim output of the double-scroll circuit (19) in $\left(p_{2}, p_{3}\right)-$ plane

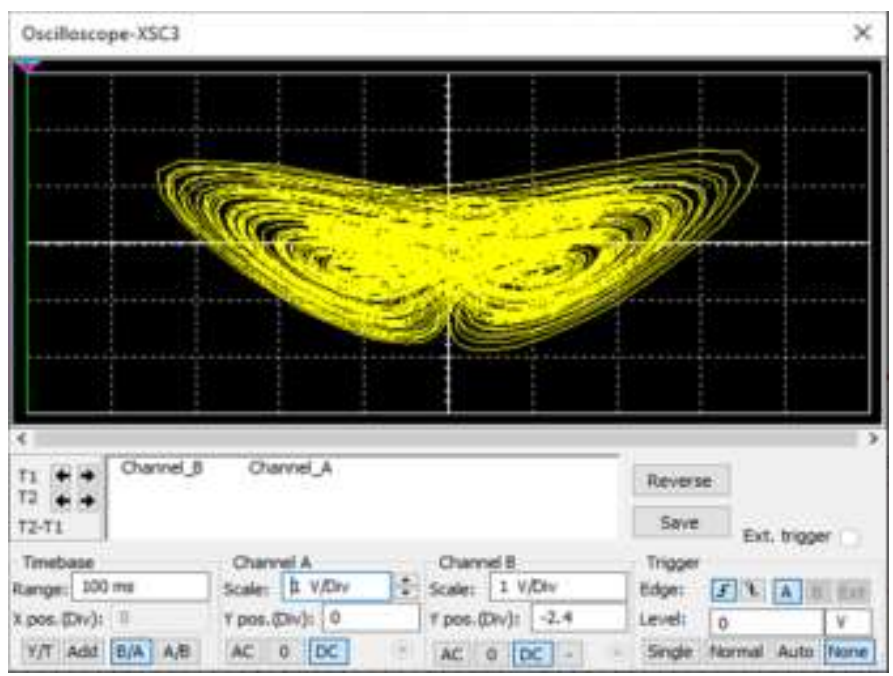

Figure 7. MultiSim output of the chaotic circuit $(19)$ in $\left(p_{1}, p_{3}\right)$-plane 


\section{CONCLUSION}

In this paper, a new multi-stable system with a double-scroll chaotic attractor is developed and detailed. Signal plots were simulated using MATLAB and multi-stability was established by showing two different coexisting double-scroll chaotic attractors for different states and same set of parameters. Using integral sliding control, synchronized chaotic attractors are achieved between drive-response chaotic attractors. A MultiSim electronic circuit was designed for the new double-scroll attractor, which is useful for practical engineering realizations.

\section{ACKNOWLEDGEMENTS}

This project is funded by the Center for Research Excellence, Incubation Management Center, Universiti Sultan Zainal Abidin.

\section{REFERENCES}

[1] S. Vaidyanathan and C. Volos, "Advances and Applications in Chaotic Systems", Berlin, Germany, Springer, 2016, doi: 10.1007/978-3-319-30279-9.

[2] J. C. Sprott, "Elegant Chaos: Algebraically Simple Chaotic Flows", Singapore, World Scientific, 2010, doi: $10.1142 / 7183$.

[3] A. Sambas, et al., "A new double-wing chaotic system with coexisting attractors and line equilibrium: Bifurcation analysis and electronic circuit simulation," IEEE Access, vol. 7, pp. 115454-115462, 2019, doi: 10.1109/ACCESS.2019.2933456.

[4] A. Sambas, et al., "A 3-D multi-stable system with a peanut-shaped equilibrium curve: Circuit design, FPGA realization, and an application to image encryption," IEEE Access, vol. 8, pp. 137116-137132, 2020, doi: 10.1109/ACCESS.2020.3011724.

[5] L. Minati, et al., "A chaotic circuit based on a physical memristor," Chaos, Solitons \& Fractals, vol. 138, p. 109990, 2020, doi: 10.1016/j.chaos.2020.109990.

[6] X. Zhang, et al., "A memristive chaotic oscillator with controllable amplitude and frequency," Chaos, Solitons \& Fractals, vol. 139, p. 110000, 2020, doi: 10.1016/j.chaos.2020.110000.

[7] B. Shi and J. Yang, "Quantification of vibration force and power flow transmission between coupled nonlinear oscillators," International Journal of Dynamics and Control, vol. 8, no. 2, pp. 418-435, 2020, doi: 10.1007/s40435019-00560-7.

[8] S. S. Saijadi, et al., "A new adaptive synchronization and hyperchaos control of a biological snap oscillator," Chaos, Solitons \& Fractals, vol. 138, art. id. 109919, 2020, doi: 10.1016/j.chaos.2020.109919.

[9] S. Vaidyanathan, "Adaptive control of the FitzHugh-Nagumo chaotic neuron model," International Journal of PharmTech Research, vol. 8, no. 6, pp. 117-127, 2015.

[10] V. T. Pham, et al., "A novel memristive neural network with hidden attractors and its circuitry implementation," Science China Technological Sciences, vol. 59, no. 3, pp. 358-363, 2016, doi: 10.1007/s11431-015-5981-2.

[11] Z. S. Al-Talib and S. F. AL-Azzawi, "Projective synchronization for 4D hyperchaotic system based on adaptive nonlinear control strategy," Indonesian Journal of Electrical Engineering and Computer Science, vol. 19, no. 2, pp. 715-722, 2020, doi: 10.11591/ijeecs.v19.i2.pp715-722.

[12] Z. S. Al-Talib and S. F. AL-Azzawi, "Projective and hybrid projective synchronization of 4-D hyperchaotic system via nonlinear controller strategy," TELKOMNIKA (Telecommunication Computing Electronics and Control), vol. 18, no. 2, pp. 1012-1020, 2020, doi: 10.11591/ijeecs.v19.i2.pp715-722.

[13] B. Sahoo and S. Poria, "The chaos and control of a food chain model supplying additional food to top-predator," Chaos, Solitons \& Fractals, vol. 58, pp. 52-64, 2014, doi: 10.1016/j.chaos.2013.11.008.

[14] M. W. Mustafa, et al., "Chaos-enhanced cuckoo search for economic dispatch with valve point effects," TELKOMNIKA (Telecommunication Computing Electronics and Control), vol. 14, no. 4, pp. 1220-1227, 2016, doi: 10.12928/telkomnika.v14i4.3473.

[15] S. Vaidyanathan and S. Rasappan, "Hybrid synchronization of hyperchaotic Qi and Lü systems by nonlinear control," Communications in Computer and Information Science, vol. 131, pp. 585-593, 2011, doi: 10.1007/978-3642-17857-3_58.

[16] S. Vaidyanathan, "A ten-term novel 4-D hyperchaotic system with three quadratic nonlinearities and its control," International Journal of Control Theory and Applications, vol. 6, no. 2, pp. 97-109, 2013.

[17] H. Medhaffar, et al., "Adaptive fuzzy control for the stabilisation of chaotic systems," International Journal of Automation and Control, vol. 14, no. 2, pp. 115-137, 2020, doi: 10.1504/IJAAC.2020.105511.

[18] A. Boubellouta and A. Boulkroune, "Intelligent fractional-order control-based projective synchronization for chaotic optical systems," Soft Computing, vol. 23, no. 14, pp. 5367-5384, 2019, doi: 10.1007/s00500-018-3490-5.

[19] S. Vaidyanathan, "Synchronization of Tokamak systems with symmetric and magnetically confined plasma via adaptive control," International Journal of ChemTech Research, vol. 8, no. 6, pp. 818-827, 2015.

[20] A. Khan and S. Kumar, "T-S fuzzy observed based design and synchronization of chaotic and hyper-chaotic dynamical systems," International Journal of Dynamics and Control, vol. 6, no. 3, pp. 1409-1419, 2018.

[21] N. M. Awal, et al., "The smallest chimera: Periodicity and chaos in a pair of coupled chemical oscillators," Chaos, vol. 29, no. 1 , art. id. 013131,2019 , doi: 10.1063/1.5060959 
[22] H. Bao et al., "Hidden bursting firings and bifurcation mechanisms in memristive neuron model with threshold electromagnetic induction," IEEE Transactions on Neural Networks and Learning Systems, vol. 31, no. 2, pp. 502511, 2020, doi: 10.1109/TNNLS.2019.2905137.

[23] Y. Deng and Y. Li, "A memristive conservative chaotic circuit consisting of a memristor and a capacitor," Chaos, vol. 30, no. 1, art. id. 013120, 2020, doi: 10.1063/1.5128384.

[24] A. Sambas, et al., "A novel chaotic system with two circles of equilibrium points: Multistability, electronic circuit and FPGA realization," Electronics, vol. 8, no. 11, 2019, art. id. 1211, 2019, doi: 10.3390/electronics8111211.

[25] A. Sambas, et al., "Investigation of chaos behavior in a new two-scroll chaotic system with four unstable equilibrium points, its synchronization via four control methods and circuit simulation," IAENG International Journal of Applied Mathematics, vol. 50, no. 1, pp. 12-21, 2020.

[26] A. Sambas, et al., "A new chaotic system with line of equilibria: dynamics, passive control and circuit design," International Journal of Electrical and Computer Engineering, vol. 9, no. 4, pp. 2365-2379, 2019, doi: 10.11591/ijece.v9i4.pp2365-2376.

[27] A. Wolf, et al., "Determining Lyapunov exponents from a time series," Physica D, vol. 16, pp. 285-317, 1985, doi: 10.1016/0167-2789(85)90011-9.

[28] J. Slotine and W. Li, “Applied Nonlinear Control”, New Jersey, USA, Prentice Hall, 2011. 